\title{
Material characterization and modeling of carbon-carbon textile composites
}

\author{
J. Vorel, J. Němeček \& M. Šejnoha \\ Department of Mechanics, Faculty of Civil Engineering, \\ Czech Technical University in Prague, Czech Republic
}

\begin{abstract}
Material modeling plays an irreplaceable role in assessing the practical applicability of any type of material system. A successful approach requires with no doubt a combination of experimental measurements with predictive numerical simulations. While numerical simulations of complex heterogeneous materials often rely on the well established concept of the statistically equivalent periodic unit cell, its formulation for complex material systems, such as carbon-carbon $(\mathrm{C} / \mathrm{C})$ composites, is far from trivial, particularly in view of a hardly negligible intrinsic porosity of these material systems. Here reliable information about the actual size, shape and distribution of pores in the composite are provided using X-Ray microtomography combined with two-dimensional images of composite sections. Similarly, difficulties arise when searching for reliable material data of individual constituents. Although usually supplied by the manufacturer, the material properties of individual constituents may significantly deviate from these values when introduced into the composite. To add credibility to numerical predictions the properties derived from nanoindentation tests performed directly on the composite are adopted. The proposed synergy of experimental and numerical modeling is exploited here to provide estimates of the elastic stiffnesses of porous multilayer $\mathrm{C} / \mathrm{C}$ composites with imperfect microstructure.
\end{abstract}

Keywords: nanoindentation, microtomography, statistically equivalent periodic unit cell, homogenization, C/C textile composites, porosity.

\section{Introduction}

While the application of PUCs in problems of strictly periodic media has a rich history, their introduction in the field of random or imperfect microstructures is 
still very much on the frontier, despite the fact that the roots for incorporating basic features of random microstructures into the formulation of a PUC were planted already in the mid 1990s [1] and extended further in [2, 3] to give rise to what we now call the concept of the Statistically Equivalent Periodic Unit Cell (SEPUC). In contrast with traditional approaches, where parameters of the unit cell model are directly measured from available material samples, the SEPUC approach is based on their statistical characterization. With regard to the present subject the interested reader is referred to [4]. A brief summary is given in Section 3.

Not only microstructural details, but also properties of individual composite constituents have a direct impact on the quality of numerical predictions. Information supplied by the manufacturer are, however, often insufficient. Moreover, the carbon matrix of the composite has properties that are dependent on particular manufacturing parameters, such as the magnitude and durations of the applied temperature and pressure. Experimental derivation of some of the parameters is therefore needed. In connection with the elastic properties of the fiber and matrix, the nanoindentation tests performed directly on the composite are discussed in Section 2 .

\section{Experimental program}

The experimental program is assumed to have the following four components:

- Two-dimensional image analysis providing binary sections of the composite further exploited in the derivation of a two-layer SEPUC. This part has been extensively studied in a number of our previous works and will not be developed here any further. Instead, the interested reader is referred to $[4,5]$.

- X-ray tomography yielding a three-dimensional map of distribution, shape and volume fraction of major pores to be introduced into, yet void-free, SEPUC.

- Nanoindentation tests supplying the local material parameters which either depend on the manufacturing process or are not disclosed by the producer.

- Laboratory evaluation of effective properties to verify predictive capabilities of numerical simulations.

For the above purposes a carbon-polymer (C/P) laminated plate was first manufactured by molding together eight layers of carbon fabric Hexcel G 1169 composed of carbon multifilament Torayca T $800 \mathrm{HB}$ and impregnated by phenolic resin Umaform LE. A set of twenty specimens having dimensions $25 \times 2.5 \times$ $2.5 \mathrm{~mm}$ were then cut out of the laminate and subjected to further treatment (carbonization $C$ at $1000^{\circ} \mathrm{C}$, reimpregnation $I$, recarbonization, second reimpregnation and final graphitization $\mathrm{G}$ at $2200^{\circ} \mathrm{C}(C I C I C G)$ ) to create the $\mathrm{C} / \mathrm{C}$ composite, see Figure 1 for an illustration and [6] for more details.

\subsection{Three-dimensional X-ray microtomography}

There is no dispute that porosity of $\mathrm{C} / \mathrm{C}$ composites plays an important role in the derivation of effective material properties. Most common approach to char- 


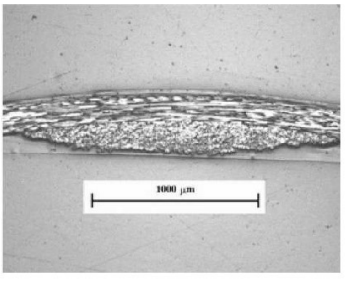

(a)

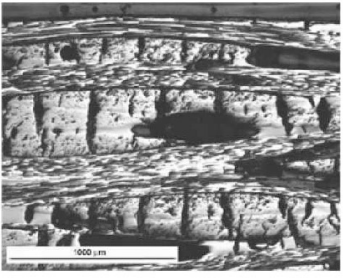

(b)

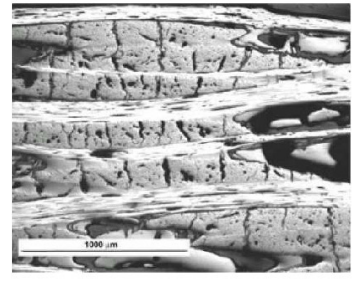

(c)

Figure 1: Examples of scanned microstructures; (a) woven fabric, (b) carbonized composite, (c) graphitized composite.

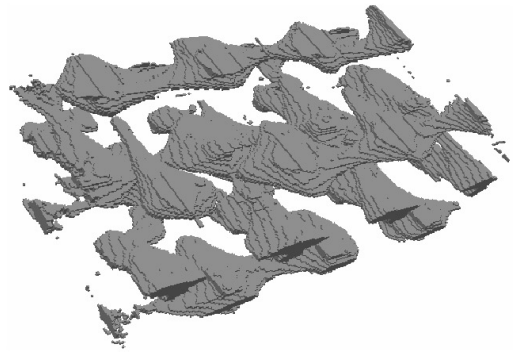

(a)

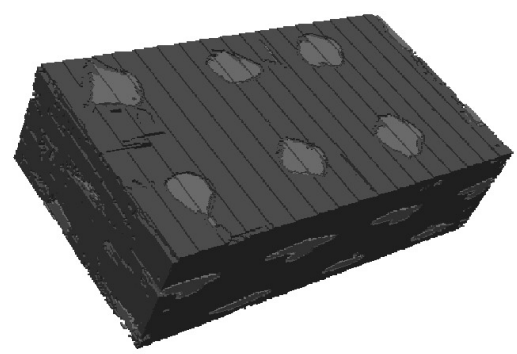

(b)

Figure 2: X-ray microtomography; (a) interior distribution and shape of large vacuoles, (b) three-dimensional view of the porous composite structure.

acterizing this property employs sectioning so that the shape of pores is estimated from $2 \mathrm{D}$ images of real $\mathrm{C} / \mathrm{C}$ composites [6] which often leads to erroneous predictions of the material response when compared to three-dimensional simulations. In this regard, the X-Ray microtomography [7] becomes a valuable tool rendering three-dimensional phase information. In the present study, high resolution computer tomography images provided by the Interfacultary Laboratory for Micro- and Nanomechanics of Biological and Biomimetical Materials of the Institute of Lightweight Design and Structural Biomechanics were used to obtain the shape, size, location and volume fraction of inter-layer (crimp) voids. A particular example of the distribution of major porosity in $\mathrm{C} / \mathrm{C}$ multi-layered plane-weave composite is presented in Figure 2. While the basic characteristics of the porosity can be extracted from these images with no particular difficulty, a direct introduction of pores in their full complexity as seen in Figure 2(a) is impossible. Instead, a discrete set of oblate spheroids approximating the shape and volume of actual pores is accommodated in between the two layers of the SEPUC fitting their true location as close as possible. 


\subsection{Phase elastic moduli from nanoindentation}

Prediction of complex macroscopic response of highly heterogeneous materials from local phase constitutive theories is a formidable aspect of micromechanical modeling. The reliability of these predictions, however, is considerably influenced by available information on material data of individual constituents. Even though supplied by the producer, these information are often insufficient for threedimensional analysis. It is also known that material properties of the matrix much depend on the fabrication of composite and may considerably deviate from those found experimentally for large unconstrained material samples.

Carbon matrix developed in the composite through a repeated process of impregnation, curing and carbonization of the phenolic resin is a solid example. This resin falls into the category of non-graphitizing resins so that the final carbon matrix essentially complies, at least in terms of its structure, with the original cross-linked polymeric precursor. Therefore, the resulting material symmetry is more or less isotropic with material parameters corresponding to those of glassy carbon. Nevertheless, when constrained the assumed matrix isotropy may evolve into the one of the fibers particularly in their vicinity. Although the PAN (polyacrylonitril) based carbon fibers are known to have a relatively low orderliness of graphen planes on nano-scale, they still posses a transverse isotropy with the value of longitudinal tensile modulus (usually available) considerably exceeding the one in the transverse direction (often lacking). Additional experiments, preferably performed directly on the composite, are therefore often needed to either validate the available local data or to derive the missing ones.

At present, nanoindentation is the only experimental technique that can be used for direct measurement of mechanical properties at material micro-level. A successful application of nanoindentation to $\mathrm{C} / \mathrm{C}$ composites has been reported in [8]. In the present study, our only attention was limited to the evaluation of the matrix elastic modulus and the transverse elastic modulus of the fiber. The remaining data were estimated from those available in the literature for similar material systems.

Three locations, distinctly separated in optic microscope, were tested - matrix, parallel fibers Figure 3(a), perpendicular fibers Figure 3(b). The matrix was therefore assumed isotropic and possible anisotropy, which may arise inside the fiber tow, was not considered. As seen in Figure 3 several measurements were recorded for each of the three locations. The measurements were performed using CSM Nanohardness tester equipped with a Berkovich tip allowing for 0.1-500 mN loading range. To ensure elastic response relatively low indentation forces up to $10 \mathrm{mN}$ were considered. The elastic moduli were extracted from an unloading part of the indentation curve. The complete set of parameters, both measured averages labeled by ${ }^{*}$ and those adopted from the literature, is available in Table 1 . Note that the matrix modulus agrees relatively well with the one found for the glassy carbon in [8].

The final note is concerned with heat treatment of the $\mathrm{C} / \mathrm{C}$ composite during fabrication. It has been observed experimentally [9] that even for T800 based composites the tensile Young's modulus increases for graphitized specimens (CICICG) 


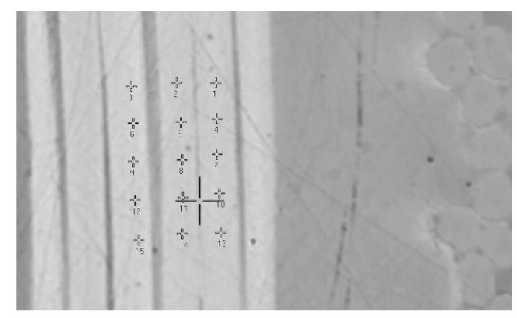

(a)

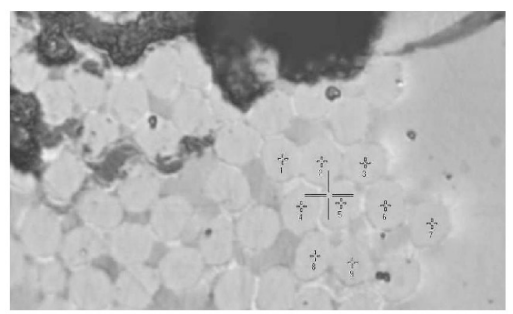

(b)

Figure 3: Nanoindentation - location of indents; (a) transverse direction, (b) longitudinal direction (compression).

when compared to only carbonized ones $(C I C I C)$. It is suggested that this phenomenon may be caused by further stiffening of carbon fibers. This, however, is difficult to address in the present study as the tensile modulus cannot be measured via nanoindentation. On the contrary, neither the matrix properties nor the porosity profile is expected to change considerably with graphitization. Therefore, the experimental data reported in [9] for the $C I C I C$ system rather than those for $C I C I C G$ system will be fostered for comparison with numerical predictions.

Table 1: Material parameters of individual phases.

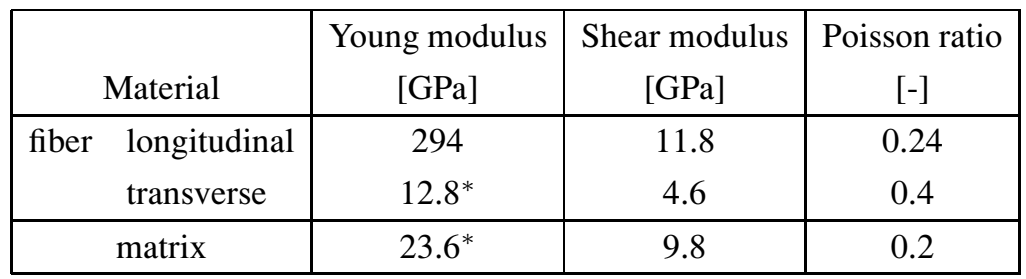

\subsection{Laboratory evaluation of effective properties}

Corroboration of a mechanics model by experimental data is still thought vital for the model to be accepted, inasmuch as there is simply nothing better, even though an experiment often comprises laboratory measurements and a theory for calculating not directly measurable parameters.

The dynamic tensile and shear moduli are typically derived from a resonant frequency method. While the basic longitudinal resonant frequency of a beam with free ends is used to extract the tensile Young's modulus $E_{11}$, the complete frequency equation for flexural vibration of a beam with free ends is solved to obtained the longitudinal shear modulus $G_{12}[10]$. 
Among other material system this technique was employed in [9] to derive the homogenized tensile and shear moduli of a four layer T800 fiber fabric based plain weave $\mathrm{C} / \mathrm{C}$ composite. As part of their study a unidirectional (UD) carbonized $C I C I C$ composite was examined. In the present study a relatively high volume fractions of fibers equal to $68 \%$ and open porosity by water penetration of $6.6 \%$ were taken from [9].

The resulting moduli stored in Table 2 (porous tow (EXP) column) were utilized here to validate the Mori-Tanaka estimates of the homogenized properties of the porous fiber tow (porous tow (MT) column). This is important as the entire three-dimensional $(6 \times 6)$ stiffness matrix of the homogenized fiber tow is needed when performing the homogenization step on meso-scale. Theoretical details and some particular applications of the MT method in the field of $\mathrm{C} / \mathrm{C}$ composites are available in $[11,12$, to cite a few $]$.

Table 2: Effective elastic properties of UD C/C composite (porous fiber tow) and $\mathrm{C} / \mathrm{C}$ laminate conductivities [9]. Young's moduli are given in [GPa].

\begin{tabular}{|c|c|c|c|}
\hline parameter & porous tow $(\mathrm{MT})$ & porous tow $(\mathrm{EXP})$ & laminate $(\mathrm{EXP})$ \\
\hline$E_{11}$ & 193.8 & $\approx 200$ & $\approx 65$ \\
$G_{12}$ & 10.3 & $\approx 11.5$ & $\approx 6$ \\
\hline
\end{tabular}

\section{Statistically equivalent period unit cell}

The concept of Statistically Equivalent Periodic Unit Cell for random or imperfect microstructures is now well established. A lucid presentation of individual steps enabling the substitution of real microstructures by their simplified artificial representatives - the SEPUCs - is available, e.g. in [2, 3, 5] and additional references given below. Herein, these steps are briefly reviewed concentrating on the specifics of multi-layer woven composites.

\subsection{Geometrical mesostructural model}

The basic building block of the adopted SEPUC is provided by a single-ply model of plain weave composite geometry proposed by Kuhn and Charalambides in [13]. The model consists of two orthogonal warp and weft tows embedded in the matrix phase and it is parametrized by four basic quantities, directly measurable by twodimensional image analysis: the half-period of tow undulation $a$, the maximal tow thickness $b$, the width of the intra-tow gap $g$ and the overall height of the ply $h$, see Figure 4(a). The three-dimensional woven composite SEPUC, shown in Figure 4(b), is formed by two identical one-layer blocks, relatively shifted by distances $\Delta_{1}, \Delta_{2}$ and $\Delta_{3}$ in the direction of the corresponding coordinate axes. 


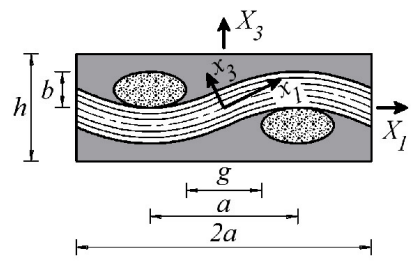

(a)

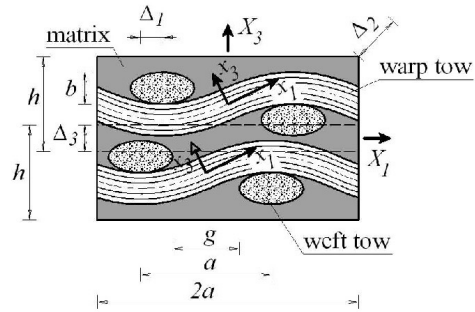

(c)

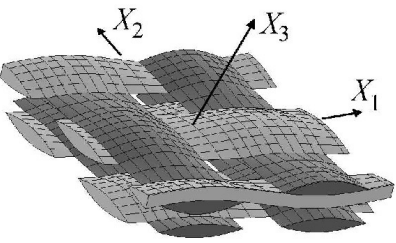

(b)

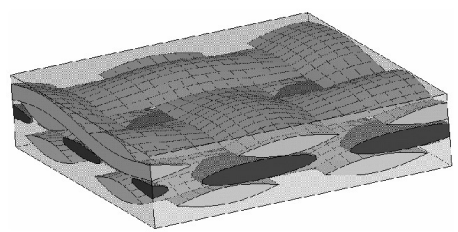

(d)

Figure 4: Geometrical model of SEPUC; (a) two-dimensional cut of a one-layer model, (b) two-layer model including periodic extension of upper layer, (c) two-dimensional cut, (d) 3D view of the geometry of a two-layer UC model with voids.

Finally, cutting a SEPUC by the plane $X_{2}=a$ or $X_{1}=a$ yields the warp or weft two-dimensional sections, used as the basis for the determination of the unit cell parameters. These are derived from the solution of an independent minimization problem discussed in details in $[3,5]$.

The porous phase is introduced next being represented by four identical oblate spheroids, the volume of which is derived from X-ray microimages shown in Figure 2. These are then periodically extended over the entire model, Figure 4(d). Their location is assumed to mimic the distribution of large vacuoles that typically appear in the location of tow crossings. However, this is difficult to achieve in general, and thereby the porous phase was excluded from the minimization problem.

\subsection{Numerical simulations}

Numerical evaluation of effective elastic moduli is presented in this section in support of the proposed concept of SEPUC in the light of multi-layered $\mathrm{C} / \mathrm{C}$ composites. The well established strategy based on the first-order homogenization approach (to cite a few [4, 5, 14]) was adopted. To that end, we consider a heterogeneous periodic unit cell $Y$ and variations of local displacement $\boldsymbol{u}(\boldsymbol{X})$ field written in terms of the uniform macroscopic quantities $\boldsymbol{E}$ as

$$
\boldsymbol{u}(\boldsymbol{X})=\boldsymbol{E} \cdot \boldsymbol{X}+\boldsymbol{u}^{*}(\boldsymbol{X})
$$


$\boldsymbol{u}^{*}$ are $Y$-periodic displacements fluctuations, respectively and $\bullet(\boldsymbol{X})$ is introduced to represent a given quantity in the global coordinate system $\boldsymbol{X}$, recall Figure 4 . Next, denoting $\mathbf{L}(\boldsymbol{x})$ the local stiffness matrix, the local microscopic constitutive equations in the local coordinate system $\boldsymbol{x}$ become

$$
\boldsymbol{\sigma}(\boldsymbol{x})=\mathbf{L}(\boldsymbol{x}) \boldsymbol{\varepsilon}(\boldsymbol{x})
$$

To complete the set of equations needed in the derivation of effective properties, we recall the Hill lemma together with Eq. (2) and write the global-local variational principles, see e.g. $[15,16$, for further details $]$ in the forms

$$
\left\langle\delta \varepsilon(\boldsymbol{x})^{\top} \mathbf{L}(\boldsymbol{x}) \boldsymbol{\varepsilon}(\boldsymbol{x})\right\rangle=0,
$$

where $\langle a(\boldsymbol{x})\rangle$ represents the volume average of a given quantity, i.e. $\langle a(\boldsymbol{x})\rangle=$ $\frac{1}{1} \int a(\boldsymbol{x}) \mathrm{d} \Omega$. In the framework of finite element approximation, the discrete forms of local gradient derived from Eq. (1) read

$$
\varepsilon(\boldsymbol{X})=\boldsymbol{E}+\mathbf{B}^{u}(\boldsymbol{X}) \boldsymbol{u}_{d}^{*}
$$

where $\mathbf{B}^{\bullet}$ stores the derivatives of the element shape functions w.r.t. $\boldsymbol{X}$ and $\boldsymbol{u}_{d}^{*}$ are the vectors of the fluctuation part of nodal displacements. Substituting Eq. (4) into Eq. (3) gives

$$
\delta \boldsymbol{u}_{d}^{* \top}\left\langle\mathbf{B}^{u}(\boldsymbol{X})^{\top} \mathbf{L}(\boldsymbol{X}) \mathbf{B}^{u}(\boldsymbol{X})\right\rangle \boldsymbol{u}_{d}^{*}=-\delta \boldsymbol{u}_{d}^{* \top}\left\langle\mathbf{B}^{u}(\boldsymbol{X})^{\top} \mathbf{L}(\boldsymbol{X})\right\rangle \boldsymbol{E},
$$

to be solved for nodal displacements $\boldsymbol{u}_{d}^{*}$. Combining Eqs. (4) and Eqs. (2) now allows us to write the volume averages of local stresses as

$$
\boldsymbol{\Sigma}=\left\langle\mathbf{T}^{\varepsilon}(\boldsymbol{X})^{\top} \boldsymbol{\sigma}(\boldsymbol{x})\right\rangle=\frac{1}{|\Omega|} \int \mathbf{T}^{\varepsilon}(\boldsymbol{X})^{\top} \mathbf{L}(\boldsymbol{x}) \mathbf{T}^{\varepsilon}(\boldsymbol{X}) \boldsymbol{\varepsilon}(\boldsymbol{X}) \mathrm{d} \Omega,
$$

also showing the relationship between material matrices in the local and global coordinate systems in terms of the transformation matrix $\mathbf{T}^{\mathcal{E}}$, see e.g. $[12,15]$,

$$
\mathbf{L}(\boldsymbol{X})=\mathbf{T}^{\varepsilon}(\boldsymbol{X})^{\top} \mathbf{L}(\boldsymbol{x}) \mathbf{T}^{\varepsilon}(\boldsymbol{X}) .
$$

The results of Eq. (6) now allows for writing the macroscopic constitutive laws as

$$
\boldsymbol{\Sigma}=\mathbf{L}^{\mathrm{H}} \boldsymbol{E}
$$

where $\mathbf{L}^{\mathrm{H}}$ is the searched homogenized effective elastic stiffness matrices.

The mapped meshing technique was employed to ensure periodicity of the finite element mesh when dicretizing the geometrical model in Figure 4(d). The results from numerical simulations as well as from available laboratory measurements are listed in Table 3. 
Table 3: The homogenized elastic moduli in [GPa] derived from the application of the two-layer SEPUC plotted in Figure 4(d).

EXPERIMET
\begin{tabular}{|cc|cccc|}
\hline$E_{11}$ & $G_{12}$ & $E_{11}$ & $G_{12}$ & $E_{33}$ & $G_{13}$ \\
\hline 65 & 6 & 60.3 & 8.1 & 11.5 & 5.3 \\
\hline
\end{tabular}

\section{Conclusion}

Evaluation of the effective properties of multilayered $\mathrm{C} / \mathrm{C}$ composites was presented. It has been shown that arriving at reliable numerical predictions requires synergy of laboratory measurements carried out on actual samples and well established and efficient homogenization techniques based on multi-scale computational strategy. Application of both the MT method on the level of fiber tow and the concept of SEPUC on the meso-scale has been justified by a reasonably close agreement between laboratory measurements and numerical predictions as also seen in Figure 5. Results derived solely from the MT method are plotted for further comparison.

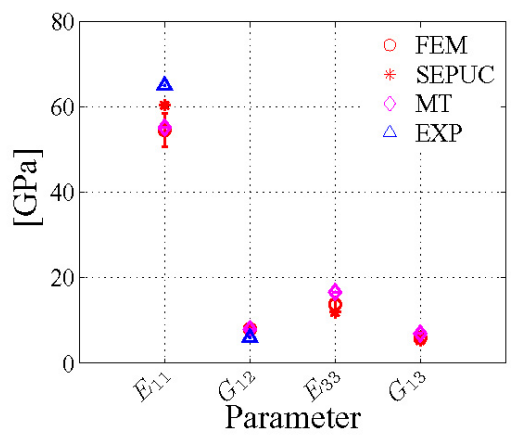

Figure 5: Comparison of the numerical and experimental results.

\section{Acknowledgements}

The financial support provided by the research project CEZ MSM 6840770003 is gratefully acknowledged. We extend our personal thanks to Dr. Dieter H. Pahr from the Technical University of Vienna for providing the X-ray images. 


\section{References}

[1] Povirk, G.L., Incorporation of microstructural information into models of two-phase materials. Acta Metallurgica et Materialia, 43(8), pp. 3199-3206, 1995.

[2] Zeman, J. \& Šejnoha, M., Numerical evaluation of effective elastic properties of graphite fiber tow impregnated by polymer matrix. Journal of the Mechanics and Physics of Solids, 49(1), pp. 69-90, 2001.

[3] Zeman, J. \& Šejnoha, M., From random microstructures to representative volume elements. Modelling and Simulation in Materials Science and Engineering, 15(4), pp. S325-S335, 2007.

[4] Vorel, J., Zeman, J., Šejnoha, M. \& Tomková, B., Homogenization plain weave composites with imperfect microstructure: Part II-Analysis of realworld materials. International Journal of Solids and Structures, 0(0), p. 0, 2010. Submitted.

[5] Zeman, J. \& Šejnoha, M., Homogenization of balanced plain weave composites with imperfect microstructure: Part I-Theoretical formulation. International Journal of Solids and Structures, 41(22-23), pp. 6549-6571, 2004.

[6] Tomková, B., Modelling of thermophysical properties of woven composites. Ph.D. thesis, TU Liberec, Czech Republic, 2006.

[7] Dobiášová, L., Starý, V., Glogar, P. \& Valvoda, V., X-ray structure analysis and elastic properties of a fabric reinforced carbon/carbon composite. Carbon, 40, pp. 1419-1426, 2002.

[8] Diss, P., Lamon, J., Carpentier, L., Loubet, J. \& Kapsa, P., Sharp indentation behavior of carbon/carbon composites and varieties of carbon. Carbon, $\mathbf{4 0 ,}$ pp. 2567-2579, 2002.

[9] Černý, M., Glogar, P. \& Machota, L., Resonant frequency study of tensile and shear elasticity moduli of carbon fibre reinforced composites (CFRC). Carbon, 38, pp. 2139-2149, 2000.

[10] Vozková, P., Multiscale modeling of mechanical properties of textile composites. Ph.D. thesis, TU Liberec, Czech Republic, 2008. In Czech.

[11] Skoček, J., Zeman, J. \& Šejnoha, M., Effective properties of Carbon-Carbon textile composites: application of the Mori-Tanaka method. Modelling and Simulation in Materials Science and Engineering, 16(8), 2008.

[12] Vorel, J. \& Šejnoha, M., Evaluation of homogenized thermal conductivities of imperfect carbon-carbon textile composites using the mori-tanaka method. Structural Engineering and Mechanics, 33(4), pp. 429-446, 2009.

[13] Kuhn, J.L. \& Charalambides, P.G., Modeling of plain weave fabric composite geometry. Journal of Composite Materials, 33(3), pp. 188-220, 1999.

[14] Kouznetsova, V., Brekelmans, W.A.M. \& Baaijens, P.T., An approach to micro-macro modeling of heterogeneous materials. Computational Mechanics, 27(1), pp. 37-48, 2001.

[15] Tomková, B., Šejnoha, M., Novák, J. \& Zeman, J., Evaluation of effective thermal conductivities of porous textile composites. International Journal for Multiscale Computational Engineering, 6(2), pp. 153-168, 2008. 
[16] Šejnoha, M. \& Zeman, J., Micromechanical modeling of imperfect textile composites. International Journal of Engineering Science, 46, pp. 513-526, 2008 . 